\title{
Progressive familial intrahepatic cholestasis types 1, 2, and 3
}

Bull LN, van Eijk MJT, Pawlikowska L, et al. A gene encoding a P-type ATPase mutated in two forms of hereditary cholestasis. Nat Genet 1998;18:219-24.

\section{Abstract}

Cholestasis, or impaired bile flow, is an important but poorly understood manifestation of liver disease. Two clinically distinct forms of inherited cholestasis, benign recurrent cholestasis (BRIC) and progressive familial intrahepatic cholestasis type 1 (PFIC1), were previously mapped to 18 q21. Haplotype analysis narrowed the candidate region for both diseases to the same interval of less than $1 \mathrm{cM}$, in which we identified a gene mutated in BRIC and PFIC1 patients. This gene (called FIC1) is the first identified human member of a recently described subfamily of P-type ATPases; ATP-dependent aminophospholipid transport is the previously described function of members of this subfamily. FIC1 is expressed in several epithelial tissues and, surprisingly, more strongly in the small intestine than in the liver. Its protein product is likely to play an essential role in enterohepatic circulation of bile acids; further characterization of FIC1 will facilitate understanding of normal bile formation and cholestasis.

de Vree JML, Jacquemin E, Strum E, et al. Mutations in the $M D R 3$ gene cause progressive familal intrahepatic cholestasis. Proc Natl Acad Sci 1998;95:282-7.

\section{Abstract}

Class III multidrug resistance (MDR) P-glycoproteins $(\mathbf{P}-\mathrm{gp}), \mathbf{m d r 2}$ in mice and MDR3 in man, mediate the translocation of phophatidylcholine across the canalicular membrane of the hepatocyte. Mice with a disrupted $\boldsymbol{m d r} \mathbf{2}$ gene completely lack biliary phopholipid excretion and develop progressive liver disease, characterized histologically by portal inflammation, proliferation of the bile duct epithelium, and fibrosis. The disease phenotype is very similar to a subtype of progressive familial intrahepatic cholestasis, hallmarked by a high serum $\gamma$-glutamyltransferase $(\gamma-G T)$ activity. We report immunohistochemistry for MDR3 P-gp, reverse transcription-coupled PCR sequence analysis, and genomic DNA analysis of MDR3 from two progressive familial intrahepatic cholestasis patients with high serum $\gamma$-GT. Canalicular staining for MDR3 P-gp was negative in the liver tissue of both patients. Reverse transcription-coupled PCR sequencing of the first patient's sequence demonstrated a homozygous 7-bp deletion, starting at codon 132 , which results in a frameshift and introduces a stop codon 29 codons downstream. The second patient is homozygous for a nonsense mutation in codon $957(\mathrm{C} \rightarrow$ T) that introduces a stop codon (TGA). Our results demonstrate that mutations in the human $M D R 3$ gene lead to progressive familial intrahepatic cholestasis with high serum $\gamma$-GT. The histopathological picture in these patients is very similar to that in the corresponding $m d r 2(-I-)$ mouse, in which mdr2 P-gp deficiency induces complete absence of phospholipid in bile.

\section{Comment}

Progressive familial intrahepatic cholestasis (PFIC) is a group of diseases characterised by cholestasis and biliary cirrhosis. Byler disease, the best known member of this group, is now also known as PFIC type 1. Byler disease is named after Jacob Byler, a farmer of Amish ancestry, who settled in Pennsylvania in the late 18th century. Patients have recurrent and later persistent cholestasis. ${ }^{1}$ The recurrent disease pattern is reminiscent of benign recurrent intrahepatic cholestasis (BRIC). In contrast to BRIC, Byler disease eventually evolves into biliary cirrhosis. Clinically the disease is characterised by jaundice, steatorrhoea, reduced growth, and a relatively low to normal serum $\gamma$-glutamyltranspeptidase $(\gamma \mathrm{GT})$ activity despite an elevated alkaline phosphatase. Positional cloning studies in a large Byler kindred revealed a mutation in a locus at chromosome 18q21-q22, the FIC1 locus. ${ }^{2-4}$ In their recent paper in Nature Genetics Bull et al suggest that this mutation affects a member of a subfamily of P-type ATPases; other members of this subfamily seem to be involved in ATP dependent aminophospholipid transport. FIC1 is expressed in several epithelial tissues and more strongly in the intestine than in the liver. Patients with this disease have high serum bile salt concentrations. Disturbed hepatobiliary (and perhaps intestinal) transport of bile salts is the most likely cause. How this relates to a P-type ATPase, possibly mediating aminophospholipid transport, is currently unknown. As expected, BRIC is genetically related to Byler disease as FIC1 is also mutated in BRIC patients, confirming earlier reports. ${ }^{4-6}$

Another form of PFIC, PFIC type 2, is characterised by persistent neonatal cholestasis with histological features of neonatal hepatitis and later biliary cirrhosis. Serum bile acid concentrations and alkaline phosphatase activities are elevated and the serum $\gamma$ GT activity is normal. Strautnieks et al suggest that the gene defect for this disease is located in a locus at chromosome $2 \mathrm{q} 24$, the FIC2 locus. ${ }^{7}$ This disease probably also results from a defect of hepatobiliary bile salt transport. A recently cloned candidate protein for the canalicular bile salt transporter belongs to the P-glycoprotein family and is called "the sister of P-glycoprotein". Interestingly this protein is encoded by a gene on chromosome $2 \mathrm{q} 24$.

de Vree and associates have characterised a third type of PFIC, PFIC type 3. This disease presents with recurrent pruritus or jaundice in the first months of life and then progresses to biliary cirrhosis. In contrast to patients with PFIC types 1 or 2, these patients have a high serum $\gamma$ GT activity. Oude Elferink's and Borst's groups have been involved in the development of the $m d r 2$ knockout mouse. ${ }^{8}$ In this model there is no phospholipid transport from liver to bile. Mdr2 in rodents, known as MDR3 in humans, has been characterised as a phospholipid translocator, mediating the transfer of phosphatidylcholine from the inner leaflet of the canalicular membrane to the outer leaflet. ${ }^{9} \mathrm{~A}$ functional bile salt transporter is needed for the transfer of phospholipids from the membrane into the bile as the extraction of phospholipids from the canalicular membrane is mediated by bile salts. ${ }^{10} M d r 2$ knockout mice produce bile in which phospholipids are low to absent and the 
bile salt concentration is normal. As reported earlier by De Leuze et al, patients with PFIC type 3 also produce bile salt containing bile without phospholipids. ${ }^{11}$ The histological abnormalities of these patients and the knockout mice are remarkably similar. They consist of periportal inflammation, extensive bile duct proliferation, feathery degeneration of hepatocytes, fibrosis and, in the knockout mice, liver malignancy. The hypothesis is that this histological picture results from the cytotoxicity of bile salts which in normal bile is antagonised by phospholipids.

The two patients described by de Vree et al lack MDR3 in the liver. RT-PCR revealed homozygous mutations in the $M D R 3$ mRNA. Genomic DNA analysis of their parents showed that they are heterozygous for this mutation. A sister of one of the patients is homozygous normal and so does not have the disease. It is interesting that the mother of one of these patients had recurrent episodes of cholestasis during pregnancy, suggesting that the heterozygous state may be associated with this condition. Neither of the patients responded to treatment with ursodeoxycholic acid (UDCA). The authors speculate that patients with the complete MDR3 defect do not respond to UDCA whereas those who do (about $50 \%$ with this disease) have a partial defect.

Will the patients or their families benefit from these findings? One positive outcome is that prenatal diagnosis in families with an affected child is now potentially possible. As for gene therapy, it is too early to speculate but a reasonable idea is that diseases which have with a recurrent disease pattern, such as PFIC-1 and PFIC-3, may be amenable to gene therapy if the functional gene can be given before the liver becomes too cirrhotic. Finally, PFIC-2 and PFIC-3 are probably true liver diseases as the proposed molecular defects affect proteins which are expressed pre- dominantly in the liver. PFIC-1 is thought to be caused by a defective protein which resides in the liver and which is even more highly expressed in the intestine. Liver transplantation in patients with PFIC-1 may only be partly corrective; persistent diarrhoea has indeed been reported in transplanted children with this disease.

P L M JANSEN M M MÜLLER

Department of Hepatology and Gastroenterology,

University Hospital Groningen,

PO Box 30.001,

9700 RB Groninigen, The Netherlands

1 Clayton RJ, Iber FL, Ruebner BH, et al. Byler disease. Fatal familial intrahepatic cholestasis in an Amish kindred. Am f Dis Child 1969;117:112-24.

2 Bull LN, Carlton VE, Stricker NL, et al. Genetic and morphological findings in progressive familial intrahepatic cholestasis (Byler disease [PFIC-1] and Byler syndrome): evidence for heterogeneity. Hepatology 1997;26:155-64.

3 Strautnieks SS, Kagalwalla AF, Tanner MS, et al. Locus heterogeneity in progressive familial intrahepatic cholestasis. f Med Genet 1996;33:833-6. Carlon VE, Knisely AS, Freimer NB. Mapping of a locus for progressive familial intrahepatic cholestasis (Byler disease) to $18 \mathrm{q} 21$-q22, the benign familial intrahepatic cholestasis (Byler disease) to 18q21-q22, the benign
recurrent intrahepatic cholestasis region. Hum Mol Genet 1995;4:1049-53.

5 recurrent intrahepatic cholestasis region. Hum Mol Genet 1995;4:1049-53. searching for shared segments: mapping a gene for benign recurrent intrahepatic cholestasis. Nat Genet 1994;8:380-6.

6 Sinke RJ, Carlton VEH, Juijn JA, et al. Benign recurrent intrahepatic cholestasis (BRIC): evidence of genetic heterogeneity and delimitation of the BRIC locus to a 7-cM interval between D18S69 and D18S64. Hum Genet 1997;100:382-7.

7 Strautnieks SS, Kagalwalla AF, Tanner MS, et al. Identification of a locus for progressive familial intrahepatic cholestasis PFIC2 on chromosome $2 \mathrm{q} 24$. Am f Hum Genet 1997;61:630-3.

8 Smit JJ, Schinkel AH, Oude Elferink RPJ, et al. Homozygous disruption of the murine mdr2 P-glycoprotein gene leads to a complete absence of phospholipid from bile and to liver disease. Cell 1993;75:451-62.

9 Crawford AR, Smith AJ, Hatch VC, et al. Hepatic secretion of phospholipid vesicles in the mouse critically depends on mdr2 or MDR3 P-glycoprotein vesicles in the mouse critically depends on mdr2 or MDR3 P-glycoprotein
expression. Visualization by electron microscopy. F Clin Invest 1997;100: 2562-7.

10 Frijters CM, Ottenhoff R, van Wijland MJ, et al. Regulation of $\mathrm{mdr} 2$ P-glycoprotein expression by bile salts. Biochem f 1997;321:389-95.

11 Deleuze JF, Jacquemin E, Dubuisson C, et al. Defect of multidrug-resistance 3 gene expression in a subtype of progressive familial intrahepatic cholestasis. Hepatology 1996;23:904-8. 